\title{
Massive Demodicosis of the Eyes in a Patient with Sjögren Syndrome: A Case Report
}

\author{
Marta Ziaja-Sołtys ${ }^{1}$ (1) $\cdot$ Magdalena Kołodziejczyk ${ }^{1} \cdot$ Beata Rymgayłło-Jankowska $^{2} \cdot$ Dominika Wróbel-Dudzińska $^{2}$. \\ Ewa Suchodoła-Ratajewicz ${ }^{3} \cdot$ Dominika Szlonzak $^{1} \cdot$ Tomasz Żarnowski $^{2} \cdot$ Anna Bogucka-Kocka $^{1}$
}

Received: 11 June 2020 / Accepted: 7 October 2020 / Published online: 31 October 2020

(c) The Author(s) 2020

\begin{abstract}
Purpose Demodex mites infestation, typically asymptomatic, is a problem for patients with weakened immune systems because it often takes the form of symptomatic, massive infection. The Demodex mites play an important role in the occurrence of a range of eye surface diseases such as Demodex blepharitis, Meibomian gland dysfunctions, conjunctivitis and corneal changes. The ocular infection is closely related to the systemic invasion. Our goal was to minimize infestation and alleviate the symptoms of massive demodicosis so as to prevent further damage to the cornea.

Methods Our research note involves a 61-year old woman diagnosed with secondary Sjögren syndrome due to rheumatoid arthritis. On the background of the autoimmune disease, corneal perforation of the left eye occurred that was cured by surgery. Then during the follow-up visit the patient was found (microscopically) massively infected with Demodex mites and the developed symptoms were particularly severe.

Results Adequate dry eye syndrome and massive demodicosis therapy significantly reduced the number of Demodex mites and improved the patient's condition.

Conclusion We would like to draw the attention of the physicians of different specialties that special care should be taken with respect to the therapy of dry eye syndrome and ocular demodicosis in patients with immunological disorders to achieve therapeutic success and avoid particularly dangerous consequences of these diseases.
\end{abstract}

Keywords Demodex ssp. · Ocular demodicosis $\cdot$ Sjögren syndrome $\cdot$ Therapy

Demodex folliculorum and Demodex brevis, of phylum Arthropods, are the most common ectoparasites specific for humans [1]. They inhabit different niches. Demodex folliculorum lives in the hair follicles, at the base of the eyelashes while Demodex brevis in the deeper layers of the skin in the sebaceous glands. Demodex spp. feeds on sebum, lymph, epithelial and gland cells. Thus both human species of Demodex occupy the areas with a high density of sebaceous

Marta Ziaja-Sołtys

marta.ziaja-soltys@umlub.pl

1 Chair and Department of Biology with Genetics, Medical University of Lublin, Witolda Chodźki 4 Street 4, 20-093 Lublin, Poland

2 Department of Diagnostics and Microsurgery of Glaucoma, Medical University of Lublin, Chmielna 1 Street, 20-079 Lublin, Poland

3 Department of General Ophthalmology, Medical University of Lublin, Chmielna 1 Street, 20-079 Lublin, Poland glands especially on the face but they can be present on the whole body [2-4]. The life cycle is simple, takes place in one host organism and lasts 15-21 days. After mating in the follicle opening, the female lays about 20 eggs inside the hair follicle or sebaceous gland. The 3-4 days later, the larvae hatch, transform to the nymphs and then into the adult forms. These changes take about 7 days $[5,6]$. The adult Demodex folliculorum can be 279-294 $\mu \mathrm{m}$ long, while the arrow-shaped egg cells reach a length of $104 \mu \mathrm{m} \times 41 \mu \mathrm{m}$. Demodex brevis is shorter, reaches a size of $165-208 \mu \mathrm{m}$, the egg cells are spindle-shaped and reach a size of $60 \mu \mathrm{m}$ $\times 34 \mu \mathrm{m}[7]$. In healthy individuals Demodex spp. are normal skin microbiota. The prevalence of healthy individuals ranges from $23-100 \%$. In the study by Wesolowska et al., the incidence of Demodex spp. infection among all participants (four groups: hospitalized patients, drug addicts, healthcare workers, medical students) was $41 \%$. The highest incidence rate was recorded among hospitalized patients and the elderly [6-9]. Demodex mites infestation, typically 
asymptomatic, is a problem for patients with weakened immune systems because it often takes the form of symptomatic, massive infection [3]. It was stated that Demodex mites keep the balance in ocular ecosystem by cleaning the lashes from bacteria, eating them, and also prevent this niche from occupying by other species of mites. Their presence influences the immune system answer to them, regulates and buffers it [2]. In the case of Demodex folliculorum over-population the cylindrical dandruff, the pathognomic symptom of infestation, is observed. The mites do not have excretory organs thus they regurgitate wastes. The epithelial cells, keratin, eggs and wastes form collars around the lashes [10]. Microabrasions, mechanical blockage of the Meibomian glands orifices caused especially by Demodex brevis mites induce epithelial hyperplasia and reactive hyperkeratynization. Chitinous exoskeleton, excreta of the mites are foreign substances for human organism and induce granulomatous reactions [11, 12].

According to the location of the parasites, two forms of demodicosis are currently known: cutaneous and ocular. Ocular demodicosis is a chronic, inflammatory disease that is underdiagnosed because of non-specific features such as itching, redness, pain, purulence, eyelashes loss or watery eyes. It is usually connected with chronic refractory blepharistis, recurrent styes, chalazion and dry eye syndrome [13].

Sjögren's syndrome (SS) is a chronic, progressive autoimmune disease characterized by lymphocytic infiltration of the exocrine glands in different sites of the organism but particularly lacrimal and salivary glands. Primary Sjögren syndrome is considered when arises alone, while secondary is associated with other underlying disease such as rheumatoid arthritis or systemic lupus erythhematosus [14, 15]. Lacrimal gland dysfunction causes dry eye symptoms that are not specific for Sjögren syndrome but is stated in 1/10 patients with that disease. Timely diagnosis and adequate therapy prevent ocular or systemic complications [16]. The dry eye symptoms of SS include itching, grittiness, soreness and also eye fatigue, reduced visual acuity, photosensitivity, ocular discharge, erythema. Accumulation of sticky mucus overnight makes difficult opening eyes in the morning. Sometimes there are more severe manifestations such as corneal ulceration, vascularization, opacification and very rarely, perforation [14]. It was stated that in patients with Sjögren syndrome Meibomian glands have increased number of occluded orifices, metaplasia and reduced quality of Meibomian gland secretions. Thus it is important to consider infestation with Demodex mites in patients who are non-responsive to Meibomian gland disease treatment $[2,17]$. Reducing the number of mites is the main and more possible to achieve the goal of treating demodicosis. It is nearly impossible to eliminate the whole population. There are different options of treatment that are individually proposed to patients depending on the severity of symptoms and numbers of mites.

Demodex are resistant to most common antiseptic substances such as $75 \%$ alcohol, $10 \%$ povidone-iodine or erythromycin. The most effective is tea tree oil with terpinen-4-ol as antimicrobial, antifungal, antiviral, antiseptic and acaricidal active agent [2].

Representative case report: 61-year-old woman applied to the Department of Ophthalmology Medical University in Lublin in November 2016 with the corneal perforation of the left eye. She had been treated for secondary Sjögren syndrome due to rheumatoid arthritis for 27 years. The left cornea perforated 18 years ago, then the patient was treated conservatively and the perforation site healed after treatment but leaving a small corneal scar. Ophthalmological examination revealed: visual acuity of the left eye $=0.1$ (without correction); visual acuity of the right eye $=0.6$ (without correction). Paracentrally towards 8.30 o' clock position perforation of the cornea of the left eye was visible. Around the perforation, there was edema and light fogging of the cornea. The anterior chamber of the left eye was significantly shallow, the lens was transparent, the details of the eye's fundus were less visible. The patient was admitted to the Clinic for conservative and surgical treatment. She received: Levofloxacinum in drops every $2 \mathrm{~h}, 1 \%$ Atropine $1 \times$ daily, artificial tears (Thealoz Duo) every $2 \mathrm{~h}$. Next day after hospital admission, the surgery was performed: closing the corneal perforation of the left eye by the amniotic membrane transplantation. After the procedure, a contact lens and punctum plugs for four lacrimal puncta were put on. The patient was discharged from the Clinic in good local and general condition on the fourth day after surgery. During the third follow-up visit in March 2017 she complained of burning, discomfort, itching and redness of the eyelid margins. The ophthalmological examination revealed: visual acuity of the left eye $=0.7$; visual acuity of the right eye $=0.9$ c.c. $+1.0 \mathrm{Dsph}$. The cornea of the left eye was smooth, the area of perforation was healed. Eyelid margins were reddened, slightly swollen. Keratin-fat cuffs suggesting a Demodex mites infestation were observed at the base of the eyelashes. After the on-site examination of eyelashes, the presence of numerous Demodex individuals were found (Fig. 1).

Four eyelashes from each eye were taken aseptically, by means of tweezers. The eyelashes were placed on a slide, soaked with a drop of $0.9 \%$ sodium-chloride solution and studied under a light microscope using a magnification of $100 \times$ and $200 \times$. On eyelashes collected from the left and right eye of patient adult Demodex individuals and a few larval forms were observed. 27 Demodex mites were found on the eyelashes of the left eye and 30 on the eyelashes of the right eye. The Demodex count was recorded as a total number of adult and larval forms that were found in all four lashes taken from each eye. Our goal was to minimize 
Fig. 1 Numerous Demodex mites on eyelashes taken from the eyes of the patient during the visit in March 2017. Many dandruff fragments are seen. Microscopic preparation in $0.9 \%$ sodium chloride solution. Magnification 200×. a Right eye; $\mathbf{b}$ Left eye

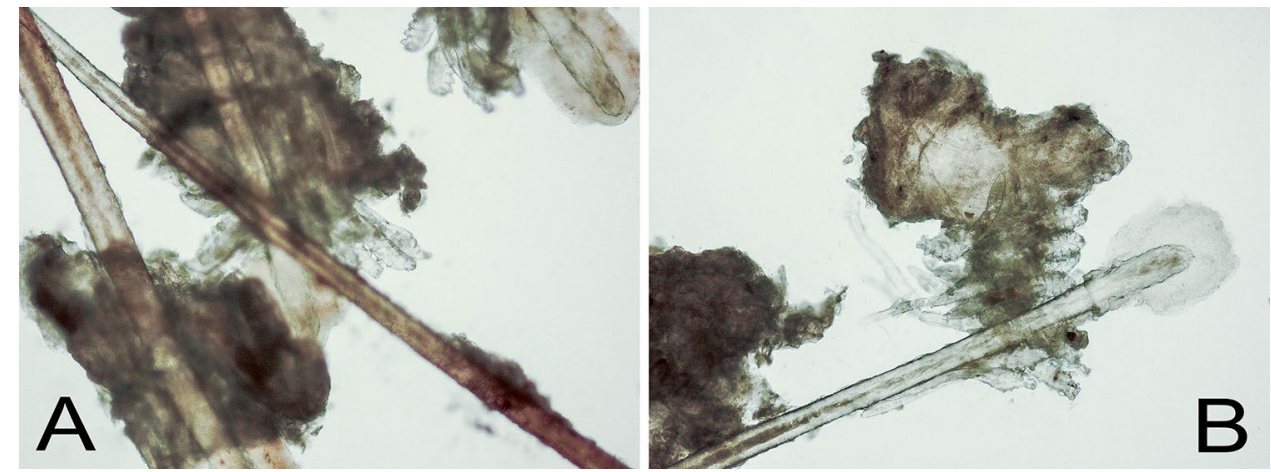

infestation and alleviate the symptoms of massive demodicosis so as to prevent further damage to the cornea. The patient received anti-Demodex treatment: Demoxoft Lipogel (Verco) on the eyelid edges with Demoxoft liquid (Verco) and Erythromycin ointment also on the eyelid edges. During subsequent control tests in May 2017, August 2017 and in November 2017, many Demodex parasites on the lashes of the patient were still present (despite the maintained anti-Demodex treatment). Moreover, in November 2017 on consultation it was stated: V.o.d. $=1.0$ c.c. $+1.0 \mathrm{Dsph}$; V.o.s. $=0.6$; Schirmer test $\mathrm{OP}=0 \mathrm{~mm}$; $\mathrm{OL}=1 \mathrm{~mm}$. There were punctum plugs for four lacrimal puncta. The next follow-up visits took place every year in April. During the last examination subjectively, the patient felt better reported less discomfort and less noticeable burning sensation. Combined dry eye treatment based on regular (5-6 times a day) artificial tears application with four punctum plugs insertion into four lacrimal puncta together with anti-Demodex treatment (Demoxoft Lipogel and Demoxoft Liquid) applying to eyelid margins, used consistently from November to April), enhanced clinical condition of the eye surface and eyelid margins. The eye surface (corneal and conjunctival surface) was moist and eyelid margins were found to be more pale. Thus the patient claimed to feel better.
According to the diminished signs and symptoms of dry eye only 4 puncta plugs for lacrimal puncta were inserted. The presence of purulent and mucous secretions in the conjunctival sac from about 4 days-the patient suffered from an infection of the upper respiratory tract and rhinitis mucosa. Regarding subject: conjunctiva was paler than before. Meibomian glands-obstructed, especially on the edge of both lower eyelids. Eyelash cuffs were still present, but in the microscopic examination of eyelashes for the presence of single mature forms of Demodex were found (in previous studies, even a dozen of individuals in various stages of development). The tea tree oil with terpinen-4-ol (the main component of Demoxoft Lipogel and Demoxoft Liquid) used regularly on eyelid margins, is very effective in diminishing the number of Demodex mites [18-20]. The purulent-mucous secretion in the conjunctival sac and internal angles of both eyes as well as redness of the eye and eyelid conjunctiva may indicate bacterial conjunctivitis probably associated with upper respiratory tract infection and rhinitis (Fig. 2).

The symptoms of ocular demodicosis and dry eye syndrome are similar except for the presence of cylindrical dandruff at the base of eyelashes that is characteristic of demodicosis. Meibomian gland dysfunction very often causes of dry
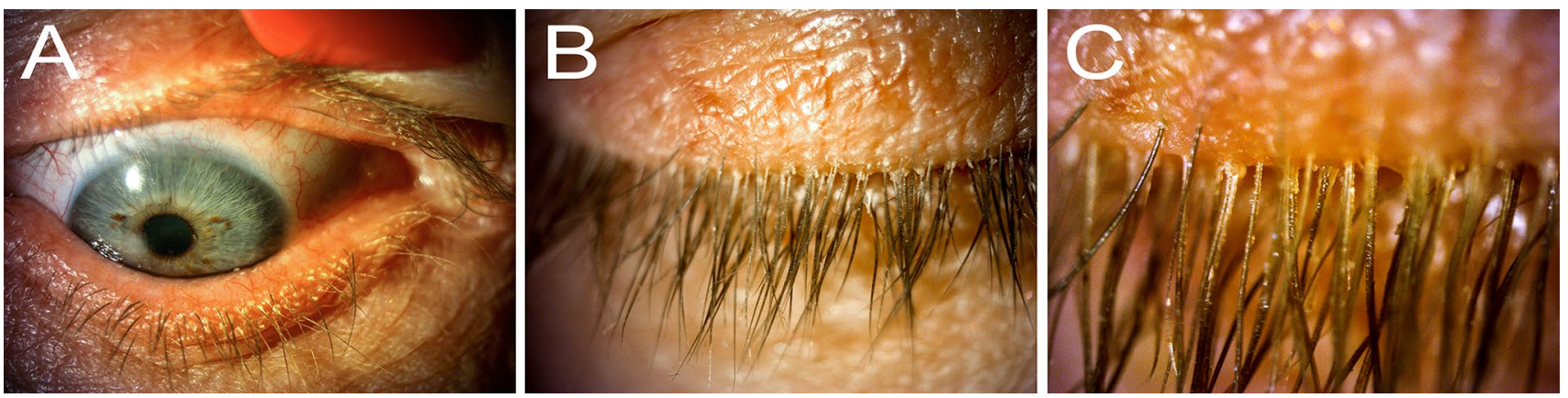

Fig. 2 a Right eye. Conjunctival hyperaemia, dilated blood vessels of the upper and lower eyelid margin, clogged Meibomian glands orifices of both eyelids, collar of tissue around the base of the eyelashes of the lower eyelid. b Upper left eyelid. Collar of tissue around the base of the eyelashes, typical for Demodex infestation. c Collar of tissue around the base of the eyelashes, typical for Demodex infestation, higher magnification 
eye syndrome. The Demodex mites are the most widespread but very often overlooked human parasites. It is known that D. folliculorum mites promote bacterial proliferation and cause inflammation of the eyelid and the conjunctiva. This enhances the production of lipases and esterases and consequently increase the viscosity and melting temperature of the meibum, reducing its secretion onto the surface of the tear film [21]. Secondary demodicosis is classified in conjunction with local or systemic immunosuppression including Sjögren's syndrome or rheumatoid arthritis [22]. Kheirkhah et al. reported corneal changes due to demodicosis. Although D. folliculorum mites seem to be more prevalent than D. bre$v i s$ they detected the last one in half of the studied cases. In addition, other authors have identified refractory and recurrent keratitis in patients with confirmed Demodex infestation. Interestingly these keratitis had been earlier treated as herpes ones, but therapy was not successful. Antiparasitic treatment with Cliradex (active compound terpinen-4-ol) has already given positive results after one week [18, 23].

Daily eyelid hygiene is the most important in the treatment of demodicosis. However, there is no standard treatment. There are many products on the pharmaceutical market, in the form of liquids, gels or wet wipes, containing extracts of tea tree and essential oils of sage or aloe. The toxic effect of these substances against Demodex was confirmed by tests. Terpinen-4-ol, an active compound isolated from tea tree oil, stimulates Demodex mites to come out to the surface of the skin, which facilitates the killing effect of the preparation against mites and their mechanical removal from the surface of the eyelids. Moreover, these preparations do not cause serious side effects, which enables their longterm use [19, 20, 23-26].

In the reported case, the positive effects of treatment, meaning reduction a number of observed Demodex mites, were obtained after the use of Demoxoft (Verco) preparations containing 4-terpinen-ol, aloe extract and Spanish sage oil. Additionally, Erythromycin ointment on the eyelids was used.

It is important that immunologists, allergists and ophthalmologists consider infestation with Demodex mites in patients who are non-responsive to Meibomian gland disease treatment with blepharitis or other infectious diseases of the ocular surface, unresolved with antiviral, antibacterial or antiallergic treatment. Massive demodicosis, chronic autoimmune diseases like Sjögren syndrome and rheumatoid arthritis are agents that favor dry eye syndrome development $[2,27,28]$.

Timely undertaking demodicosis therapy suppresses effects of parasitic activity on the host organism that cause direct damage to the eyelid edges, acute inflammatory reaction and Meibomian glands dysfunctions. As Demodex are the vectors for bacteria, viruses and fungi the therapy breaks their transmission path. The case we report confirms that special care should be taken by the physicians with respect to the therapy of dry eye syndrome and ocular demodicosis in patients with Sjögren syndrome to achieve therapeutic success and avoid particularly dangerous consequences of these diseases.

Author contributions All authors contributed to the study conception and design. Material preparation, data collection and analysis were performed by Beata Rymgayłło-Jankowska, Ewa Ratajewicz-Suchodoła and Marta Ziaja-Sołtys. The first draft of the manuscript was written by Marta Ziaja-Sołtys, Beata Rymgayłło-Jankowska and all authors commented on previous versions of the manuscript. Writing-review, editing: Marta Ziaja-Sołtys. Supervision: Tomasz Żarnowski, Anna Bogucka-Kocka. All authors read and approved the final manuscript.

Funding This study was supported by the Statutory Funds of the Medical University of Lublin No. DS43 (Anna Bogucka-Kocka), provided by the Polish Ministry of Science and Higher Education.

\section{Compliance with ethical standards}

Conflict of interest The authors declare that they have no conflict of interest.

Ethics approval Protocol for use human tissues in research was approved by Bioethical Commission, Medical University of Lublin, Poland (Permission No. KE-0254/265/216). The patient consented to anonymous use of the examinations results for scientific publication.

Open Access This article is licensed under a Creative Commons Attribution 4.0 International License, which permits use, sharing, adaptation, distribution and reproduction in any medium or format, as long as you give appropriate credit to the original author(s) and the source, provide a link to the Creative Commons licence, and indicate if changes were made. The images or other third party material in this article are included in the article's Creative Commons licence, unless indicated otherwise in a credit line to the material. If material is not included in the article's Creative Commons licence and your intended use is not permitted by statutory regulation or exceeds the permitted use, you will need to obtain permission directly from the copyright holder. To view a copy of this licence, visit http://creativecommons.org/licenses/by/4.0/.

\section{References}

1. Sarac G (2019) A comparison of the efficacy and tolerability of topical agents used in facial Demodex treatment. J Cosmet Dermatol 2019:1-4. https://doi.org/10.1111/jocd.12986

2. Fromstein SR, Harthan JS, Patel J, Opitz DL (2018) Demodex blepharitis: clinical perspectives. Clin Optom (Auckl) 2018:5763. https://doi.org/10.2147/OPTO.S142708

3. Forton FMN, De Maertelaer V (2017) Two consecutive standardized skin surface biopsies: an improved sampling method to evaluate Demodex density as a diagnostic tool for rosacea and demodicosis. Acta Derm Venereol 97:242-248. https://doi. org/10.2340/00015555-2528

4. Rusiecka-Ziółkowska J, Nokiel M, Fleischer M (2013) Demodex - an old pathogen or a new one? Adv Clin Exp 23(2):295298. https://doi.org/10.17219/acem/37081 
5. Marcinowska Z, Kosik-Bogacka D, Łanocha-Arendarczyk N, Czepita D, Łanocha A (2015) Demodex folliculorum i Demodex brevis. Pom J Life Sci 61(1):108-114. https://pdfs.semanticscholar .org/1878/21271a62f9f75516b6721d9dff5c18c6f47f.pdf

6. Rather PA, Hassan I (2014) Human Demodex Mite: the versatile mite of dermatological importance. Indian J Dermatol 59(1):6066. https://doi.org/10.4103/0019-5154.123498

7. Wesołowska M, Knysz B, Reich A, Blazejewska D, Czarnecki M, Gladysz A, Pozowski A, Misiuk-Hojlo M (2014) Prevalence of Demodex spp. in eyelash follicles in different populations. Arch Med Sci 2014:319-324. https://doi.org/10.5114/aoms.2014.42585

8. Norn MS (1971) Demodex folliculorum. Incidence, regional distribution, pathogenicity. Dan Med Bull 18:14-17. https://pubme d.ncbi.nlm.nih.gov/5110952/

9. Rufli T, Mumcuoglu Y (1981) The hair follicle mites Demodex folliculorum and Demodex brevis: biology and medical importance. A review. Dermatologica 162:1-11. https://doi.org/10.1159/00025 0228

10. Rabensteiner DF, Aminfar H, Boldin I, Nitsche-Resch M, Berisha B, Schwantzer G, Horwath-Winter J (2019) Demodex mite infestation and its associations with tear film and ocular surface parameters in patients with ocular discomfort. Am J Ophthalmol 204:7-12. https://doi.org/10.1016/j.ajo.2019.03.007

11. Luo X, Li J, Chen Ch, Tseng S, Liang L (2017) Ocular demodicosis as potential cause of ocular surface inflammation. Cornea 36:9-14. https://doi.org/10.1097/ICO.0000000000001361

12. Murphy O, O'Dwyer V, Lloyd-McKernan A (2019) Ocular Demodex folliculorum: prevalence and associated symptoms in an Irish population. Int Ophtalmol 39(2):405-417. https://doi.org/10.1007/ s10792-018-0826-1

13. Sędzikowska A, Oseka M, Skopiński P (2018) The impact of age, sex, blepharitis, rosacea and rheumatoid arthritis on Demodex mite infection. Arch Med Sci 14(2):353-356. https://doi. org/10.5114/aoms.2016.60663

14. Beckman KA, Luchs J, Milner MS, Ambrus JL Jr (2017) The potential role for early biomarker testing as part of a modern, multidisciplinary approach to Sjögren's syndrome diagnosis. Adv Ther 2017:799-812. https://doi.org/10.1007/s12325-017-0501-3

15. Venables PJ (2004) Sjögren's syndrome. Best Pract Res Clin Rheumatol 18(3):313-329. https://doi.org/10.1016/j.berh.2004.02.010

16. Karakus S, Baer AN, Akpek EK (2019) Clinical correlations of novel autoantibodies in patients with dry eye. J Immunol Res 2019:7935451. https://doi.org/10.1155/2019/7935451

17. Choi W, Ha JY, Li Y, Choi JH, Ji YS, Yoon KC (2019) Comparison of the meibomian gland dysfunction in patients with chronic ocular graft-versus-host disease and Sjögren's syndrome. Int J Ophthalmol 12(3):393-400. https://doi.org/10.18240 /ijo.2019.03.07

18. Kheirkhah A, Casas V, Li W, Raju V, Tseng SCG (2007) Corneal manifestations of ocular demodex infestation. Am J Ophtalol 143(5):743-749. https://doi.org/10.1016/j.ajo.2007.01.054

19. Wasyluk J, Krajewska M, Czarzasta K (2018) Ocular demodicosis-a contemporary clinical condition. Ophtha Therapy. 5(1):2125. https://doi.org/10.24292/01.OT.sup1310518.05

20. Krajewska M, Wasyluk J, Sędzikowska A et al (2013) Evaluation of the effectiveness and safety of Demoxoft Lipogel and Demoxoft preparations used in patients with Demodex blepharitis-preliminary results. Ophthalmology 2013:9-11. https://www.researchga te.net/publication/259928469

21. Baudouin C, Messmer EM, Aragona P et al (2016) Revisiting the vicious circle of dry eye disease: a focus on the pathophysiology of meibomian gland dysfunction. Br J Ophthalmol 100:300-306. https://doi.org/10.1136/bjophthalmol-2015-307415

22. Formentini Scotton Jorge M, Zanardi Miguel LM, Santos BC, Vilaverde Schmitt J (2018) Demodicosis as treatment complication of amicrobial pustulosis of the folds. An Bras Dermatol 93(4):566-569. https://doi.org/10.1590/abd1806-4841.20187171

23. Karakurt Y, Zeytun E (2018) Evaluation of the efficacy of the tea tree oil on the density of Demodex mites (Acari: Demodicidae) and ocular symptoms in patients with demodectic blepharitis. J Parasitol 104(5):473-478. https://doi.org/10.1645/18-46

24. Tighe S, Gao YY, Tseng SC (2013) Terpinen-4-ol is the most active ingredient of tea tree oil to kill demodex mites. Transl Vis Sci Technol 2(7):2. https://doi.org/10.1167/tvst.2.7.2

25. Lam NSK, Long XX, Griffin RC et al (2018) Can the tea tree oil (Australian native plant: Melaleuca alternifolia Cheel) be an alternative treatment for human demodicosis on skin? Parasitology 2018:1-11. https://doi.org/10.1017/S0031182018000495

26. Gao YY, Di Pascuale MA, Elizondo A et al (2007) Clinical treatment of ocular demodecosis by lid scrub with tea tree oil. Cornea 26(2):136-143. https://doi.org/10.1097/01.ico.0000244870.62384 .79

27. Moris García V, Valenzuela Vargas G, Marín Cornuy M, Aguila Torres P (2019) Ocular demodicosis: a review. Arch Soc Española Oftalmol (Engl Ed) 94(7):316-332. https://doi.org/10.1016/j.oftal .2019 .04 .003

28. Wu M, Wang X, Han J, Shao T, Wang Y (2019) Evaluation of the ocular surface characteristics and Demodex infestation in pediatric and adult blepharokeratoconjunctivitis. BMC Ophthalmol 19:67. https://doi.org/10.1186/s12886-019-1074-5 